\title{
Luteolin acts as a radiosensitizer in non-small cell lung cancer cells by enhancing apoptotic cell death through activation of a $\mathrm{p38} / \mathrm{ROS} / \mathrm{caspase}$ cascade
}

\author{
HYUN-JI CHO ${ }^{1}$, KWANG-CHUL AHN ${ }^{1}$, JAE YEON CHOI ${ }^{1}$, SANG-GU HWANG ${ }^{1}$, \\ WUN-JAE KIM ${ }^{2}$, HONG-DUCK $\mathrm{UM}^{1}$ and JONG KUK PARK ${ }^{1}$ \\ ${ }^{1}$ Department of Radiation Cancer Research, Korea Institute of Radiological and Medical Sciences, \\ Seoul 139-706; ${ }^{2}$ Department of Urology, College of Medicine, Chungbuk National \\ University, Cheongju, Chungbuk 361-763, Republic of Korea
}

Received November 7, 2014; Accepted December 9, 2014

DOI: 10.3892/ijo.2015.2831

\begin{abstract}
To improve radiation therapy, the development of effective radiosensitizer is required. Fifty percent inhibitory concentration $\left(\mathrm{IC}_{50}\right)$ values of $3^{\prime}, 4^{\prime}, 5^{\prime}, 7^{\prime}$-tetrahydroxyflavone (luteolin) against NCI-H460 and -H1299 non-small cell lung cancer (NSCLC) cells were determined using 3-(4,5-dimethylthiazol-2-yl)-2,5-diphenyltetrazolium bromide (MTT) assays. Radiosensitizing activity was assessed in vitro by treating cells with luteolin prior to irradiation of $\gamma$-ionizing radiation (IR), and performing cell count and clonogenic assays. Cell signaling pathways involved in the radiosensitizing effects of luteolin were examined using propidium iodide (PI) uptake, reactive oxygen species (ROS) detection and immunoblot assays, with or without specific chemical inhibitors. Apoptotic cell death was confirmed by PI uptake and immunoblot assays. In vivo radiosensitizing activity was tested using an NCI-H460 cell xenograft model in nude mice. Tumor size was measured and apoptosis was determined with terminal deoxynucleotidyl transferase dUTP nick end labeling (TUNEL) assay in animals in four treatment groups: mock-treated control, luteolin only, IR only, and luteolin plus IR. Treatment with luteolin or IR induced NSCLC cell death in vitro, but the combination of luteolin pre-treatment and IR was more effective than either agent alone, yielding dose enhancement ratios (DERs) of 1.22 and 1.35 for NCI-H460 and -H1299 cells, respectively. Combined treatment with luteolin and IR enhanced apoptotic cell death in association with downregulation of B-cell lymphoma 2 (Bcl-2) and activation of caspase- $3,-8$, and -9 ; it
\end{abstract}

Correspondence to: Dr Jong Kuk Park, Department of Radiation Cancer Research, Korea Institute of Radiological and Medical Sciences, 215-4 Gongneung-dong, Nowon-gu, Seoul 139-706, Republic of Korea

E-mail: jkpark@kirams.re.kr

Key words: radiosensitizer, luteolin, apoptosis, radiation, lung cancer, ROS, p38 MAPK, caspase also induced phosphorylation of $\mathrm{p} 38$ mitogen-activated protein kinase (MAPK) and ROS accumulation. Inhibition of p38 MAPK decreased ROS production, and inhibition of either p38 MAPK or ROS production attenuated apoptotic cell death and activation of caspase-8 and -9. In a xenograft model, tumor growth was delayed by 21.8 days in the luteolin/IR combination group compared with controls, and apoptotic cell death was increased. The enhancement factor of the luteolin and IR combination was 1.83 . Collectively, these findings indicate that luteolin acts as a radiosensitizer by enhancing apoptotic cell death through activation of a p38/ROS/caspase cascade.

\section{Introduction}

Lung cancer is among the most lethal diseases worldwide. For non-small cell lung cancer (NSCLC) in particular, the 5-year survival rate is very low (1). Three main therapeutic modalities are used to treat NSCLC: surgery, radio- and chemotherapy. Both radio- and chemotherapy often evoke therapeutic resistance, which is a major obstacle encountered during the treatment of all types of cancer, including NSCLC. One approach that is being used to enhance therapeutic efficacy and improve cancer patient survival in a variety of cancer settings is combination treatment with anticancer drugs and radiotherapy. This approach is based on the premise that anticancer drugs act via a different mechanism than radiotherapy and, importantly, may enhance the sensitivity of the cancer to the effects of ionizing radiation (IR). Among existing anticancer drugs that have been used as radiotherapy-enhancing agents, or 'radiosensitizers', are non-steroidal anti-inflammatory drugs, 5-fluorouracil, paclitaxel (and related taxane derivatives), and gemcitabine (2-7). Although many chemo-radiotherapy trials have been conducted using well-known anticancer drugs, developing new radiosensitizers that are more effective and less toxic remains an important priority (8-10).

With the advent of molecular biology has come the identification of new molecular markers of cancer with the potential to serve as targets of therapeutic drug candidates (11). There is considerable research interest in these markers, with both academic and industry researchers seeking to develop novel, 
targeted drugs or therapeutic antibodies for personalized therapy. Likewise, research on radiosensitizer development has also focused on developing targeted agents (8). But the development of novel therapeutic reagents is a costly and time-consuming process; therefore, conventional agents with confirmed biological safety or that have shown anticancer effects are also receiving renewed attention $(12,13)$.

$3^{\prime}, 4^{\prime}, 5^{\prime}, 7^{\prime}-$ Tetrahydroxyflavone (luteolin) is a flavonoid isolated from various plants, including edible and traditional medicinal plants. Flavonoids are secondary metabolites of plants characterized by their diphenylpropane structure (C6-C3-C6). Numerous investigations have sought to identify links between the consumption of food containing high concentrations of luteolin and beneficial effects on several chronic diseases, but have been unable to confirm a correlation. Although physiological effects of luteolin in foods have not been established, purified luteolin and several of its derivatives have been shown to exhibit various, significant biological effects, including antioxidant, anti-inflammatory, antimicrobial, and cancer chemotherapeutic/chemoprevention activity, among others (14). The anticancer effects of luteolin, in particular, have been actively investigated. These studies have reported that luteolin modulates various aspects of the cancer cell machinery, inhibiting CDK2 and causing cell cycle arrest in G0/G1, disrupting cellular homeostasis by depleting ATP and inhibiting glucose uptake, and inducing apoptosis through activation of caspases or promotion of mitochondrial dysfunction by pro-apoptotic B-cell lymphoma 2 (Bcl-2) proteins, among other actions. Moreover, numerous studies have shown that luteolin suppresses the growth of cancer cell lines in vitro as well as cancer xenografts in vivo (15).

In this study, we examined the anticancer effects of luteolin and determined its 50\% inhibitory concentration $\left(\mathrm{IC}_{50}\right)$ values against NSCLC cell lines. We also assessed the effects of combined treatment with luteolin and IR on $\mathrm{IC}_{50}$ values and demonstrated that combination treatment enhanced apoptotic cell death in vitro and in vivo through activation of a p38/ROS/caspase cascade.

\section{Materials and methods}

Cell culture and chemicals. The human NSCLC cell lines, NCI-H1299 and -H460, were purchased from American Type Culture Collection (Rockville, MD, USA). SB203580, N-acetyl-L-cysteine (NAC), 2',7'-dichlorofluorescin diacetate (DCF-DA) and carbobenzoxy-valyl-alanyl-aspartyl-[O-methyl]-fluoromethylketone (z-VAD-fmk) were obtained from Calbiochem (La Jolla, CA, USA). Luteolin was purchased from Sigma-Aldrich (St. Louis, MO, USA).

Immunoblot analysis. NCI-H1299 and -H460 cells were seeded in 60-mm cell culture dishes and treated under various experimental conditions. Treated cells were trypsinized, washed with ice-cold phosphate-buffered saline (PBS), and collected by centrifugation. Whole-cell lysates were prepared from harvested cells by incubating cell pellets in RIPA buffer [50 mmol/1 Tris pH 8.0, $150 \mathrm{mmol} / 1 \mathrm{NaCl}, 1 \% \mathrm{NP}-40,0.5 \%$ deoxycholic acid, and $0.1 \%$ sodium dodecyl sulfate (SDS)] containing a protease and phosphatase inhibitor cocktail (Sigma-Aldrich). Proteins in cell lysates were separated
SDS-polyacrylamide gel electrophoresis (PAGE) on 12\% gels and transferred to nitrocellulose membranes (Invitrogen Life Technologies, Carlsbad, CA, USA). Membranes were incubated with primary antibodies against caspase-3, -8 and -9 , Bcl-2, phospho-p38, and p38 (Cell Signaling Technology, Inc., Beverly, MA, USA). An anti- $\beta$-actin antibody (Santa Cruz Biotechnology, Inc., Santa Cruz, CA, USA) was used as a control for equal loading. After washing with PBS/Tween-20 (PBST), membranes were incubated with the appropriate secondary antibody. Immunoreactive proteins were detected using a chemiluminescence kit (Thermo Fisher Scientific, Inc., Rockford, IL, USA). Relative band densities of target proteins, determined densitometrically and normalized to those of $\beta$-actin in each experiment, were analyzed using ImageJ software (NIH, Bethesda, MD, USA).

MTT assay and $\mathrm{IC}_{50}$ determination. NCI-H460 and -H1299 cells were seeded onto 96 -well plates $\left(4 \times 10^{3}\right.$ cells/well) and treated with different concentrations $(10,20,30,40,50$ and $100 \mu \mathrm{M})$ of luteolin. After incubating for $72 \mathrm{~h}, 50 \mu \mathrm{l}$ of 3-(4,5-dimethylthiazol-2-yl)-2,5-diphenyltetrazolium bromide (MTT) solution $(2 \mathrm{mg} / \mathrm{ml})$ were added to each well and the plates were incubated for additional $2 \mathrm{~h}$ at $37^{\circ} \mathrm{C}$. Dark-blue formazan crystals generated by the activity of live cells were dissolved in $150 \mu \mathrm{l}$ of dimethyl sulfoxide (DMSO), and the absorbance of individual wells at $545 \mathrm{~nm}$ was determined using a microplate reader (Original Multiskan; Thermo Fisher Scientific, Inc., Waltham, MA, USA). IC $_{50}$ values were calculated from a concentration-response analysis performed using SoftMax Pro (Molecular Devices, Sunnyvale, CA, USA).

Clonogenic assay. NCI-H460 and -H1299 cells were seeded in triplicate $60-\mathrm{mm}$ dishes at cell concentrations estimated to yield 20-100 colonies/dish (100, 200, 400, 600 and 1,000 cells/dish). After $24 \mathrm{~h}$ of incubation, NCI-H460 and -H1299 cells were pre-treated with 20 or $30 \mu \mathrm{M}$ luteolin for $6 \mathrm{~h}$ and then exposed to different doses of IR $(1,3,5$, or $7 \mathrm{~Gy})$ using ${ }^{137} \mathrm{Cs}$ as a radiation source [Atomic Energy of Canada Limited (AECL), Mississauga, ON, Canada]. Cells were cultured for 10-14 days, and colonies $>200 \mu \mathrm{m}$ in diameter were counted using a colony counter (Imaging Products International, Inc., Chantilly, VA, USA). Dose enhancement ratios (DERs) were calculated as described previously (16).

Cell counting assay. Cells were seeded at a density of $1 \times 10^{5}$ cells in $60-\mathrm{mm}$ dishes, and treated with luteolin $(20$ or $30 \mu \mathrm{M})$ or left untreated. After $6 \mathrm{~h}$, cells were exposed to IR (2 or $3 \mathrm{~Gy})$ and incubated for $72 \mathrm{~h}$. Thereafter, the number of cells in each group was determined by counting under a microscope using a hemocytometer.

Propidium iodide uptake assay. Propidium iodide (PI) (Sigma-Aldrich) was used to detect apoptotic cell death. Cells were seeded at a density of $1 \times 10^{5}$ cells/well in 6 -well plates and then pre-incubated with or without luteolin for $6 \mathrm{~h}$. Cells were then exposed to IR ( 2 or $3 \mathrm{~Gy}$ ) and incubated for $72 \mathrm{~h}$. Cells were then washed twice with cold PBS and resuspended in $200 \mu \mathrm{l}$ of $5 \mu \mathrm{g} / \mathrm{ml}$ PI solution. Apoptosis was detected and analyzed using a FACSort flow cytometer (Becton-Dickinson, Franklin Lakes, NJ, USA). 
A<smiles>O=c1cc(-c2ccc(O)c(O)c2)oc2cc(O)cc(O)c12</smiles>

B

NCl-H460

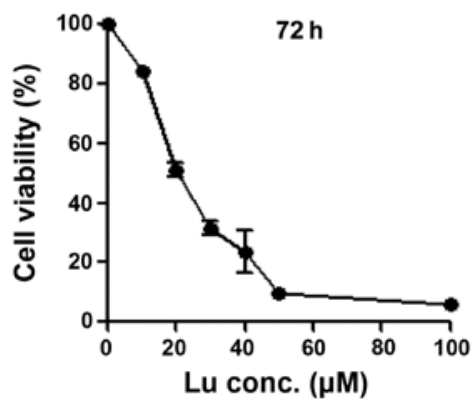

$\mathrm{NCl}-\mathrm{H} 1299$

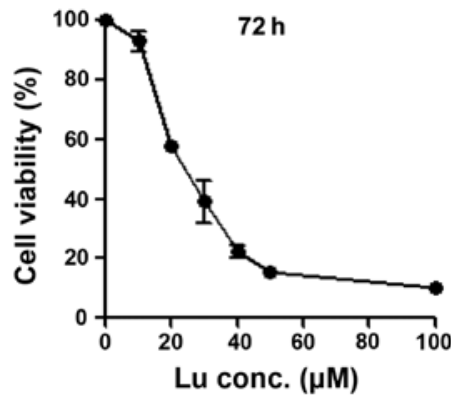

Figure 1.3',4',5',7'-Tetrahydroxyflavone (luteolin) induces non-small cell lung cancer (NSCLC) cell death. (A) Molecular structure of luteolin. (B) Determination of 50\% inhibitory concentration $\left(\mathrm{IC}_{50}\right)$ values for luteolin against NCI-H460 and -H1299 cells by 3-(4,5-dimethylthiazol-2-yl)-2,5-diphenyltetrazolium bromide (MTT) assay. MTT assays were performed during $72 \mathrm{~h}$.

Xenograft size determination and TUNEL assay. A xenograft model for evaluating the in vivo effect of luteolin was created by injecting 6-week-old BALB/cAnNCrj-nu/nu strain mice (Charles River Laboratories Japan, Inc., Kanagawa, Japan) with NCI-H460 cells $\left(1 \times 10^{7}\right)$. Mice were divided into four groups (5 mice/group): control (mock treated), IR only, luteolin only, and luteolin and IR (combination treatment). When xenografts reached $\sim 100-120 \mathrm{~mm}^{3}$, mice in luteolin only and combination treatment groups were subcutaneously injected with $10 \mathrm{mg} / \mathrm{kg}$ of luteolin; for IR-only and control groups, mice were injected with an equal volume of vehicle solution (DMSO). After $6 \mathrm{~h}$, IR-only and combination treatment groups were irradiated with $5 \mathrm{~Gy}$. This protocol was repeated three times at 5-day intervals for 35 days. Tumor dimensions (long and short axis) were detected over 35 days and tumor volumes were calculated as (short $\mathrm{axis}^{2} \mathrm{x}$ long axis)/2. For irradiation, mice were anesthetized by intraperitoneal injection of $100 \mu \mathrm{l}$ of Zoletil (Virbac Laboratories, Carros, France), then fixed to an acrylic plate and locally irradiated with a ${ }^{60} \mathrm{Co} \gamma$-ray source (Theratrom 780; AECL). Body parts other than tumor xenografts were protected with lead blocks. For terminal deoxynucleotidyl transferase dUTP nick end labeling (TUNEL) assays, xenografts were extracted, fixed with formaldehyde, and then embedded in a paraffin block. Sliced tissues were stained and analyzed with an ApopTag TUNEL assay kit (Merck KGaA, Darmstadt, Germany) as described by the manufacturer. Tumor growth delay values were calculated as described in Table I.

ROS detection assay. Reactive oxygen species (ROS) detection assays were performed as described previously (17). Cells were seeded at a density of $1 \times 10^{5}$ cells/well in 6 -well plates and pre-incubated with or without luteolin for $6 \mathrm{~h}$. Cells in IR-only and combination treatment groups were then exposed to 2 Gy of IR, with or without pharmacological inhibitors.
Table I. Analysis of tumor growth delay.

\begin{tabular}{lcc}
\hline Treatment & Days $^{\mathrm{a}}$ & Growth delay $^{\mathrm{b}}$ \\
\hline Control & 7.8 & \\
Luteolin only & 12 & 4.2 \\
IR only & 17.4 & 9.6 \\
Luteolin + IR & 29.6 & 21.8 \\
Enhancement factor $^{\mathrm{c}}$ & 1.83 & \\
\hline
\end{tabular}

${ }^{\mathrm{a}}$ Time required for tumors to reach a volume of $1,500 \mathrm{~mm}^{3} .{ }^{\mathrm{b}}$ Additional time required for tumors to reach a volume of $1,500 \mathrm{~mm}^{3}$ compared with control. ${ }^{\circ}$ Calculated as (growth delay for combination - growth delay for luteolin only)/growth delay for IR only. Luteolin, 3', 4',5',7'-tetrahydroxyflavone; IR, ionizing radiation.

After treating for $24 \mathrm{~h}$, cells were trypsinized and incubated with $20 \mu \mathrm{M}$ DCF-DA for $5 \mathrm{~min}$. ROS were detected and analyzed using a FACSort flow cytometer (Becton-Dickinson).

Statistical analysis. Data were analyzed using GraphPad Prism software (GraphPad Software, Inc., La Jolla, CA, USA), and the significance of differences between experimental groups was determined using Student's t-test. $P<0.05$ was considered significant; individual $\mathrm{p}$-values are denoted by asterisks in figures $(p<0.05,0.01$ and 0.001$)$. The number above each point or bar in every graph indicates the mean percentage of three independent experiments, and error bars signify standard deviation (SD).

\section{Results}

Combined treatment with luteolin and IR enhances cell death. Luteolin (Fig. 1A) is considered an anticancer drug candidate. 

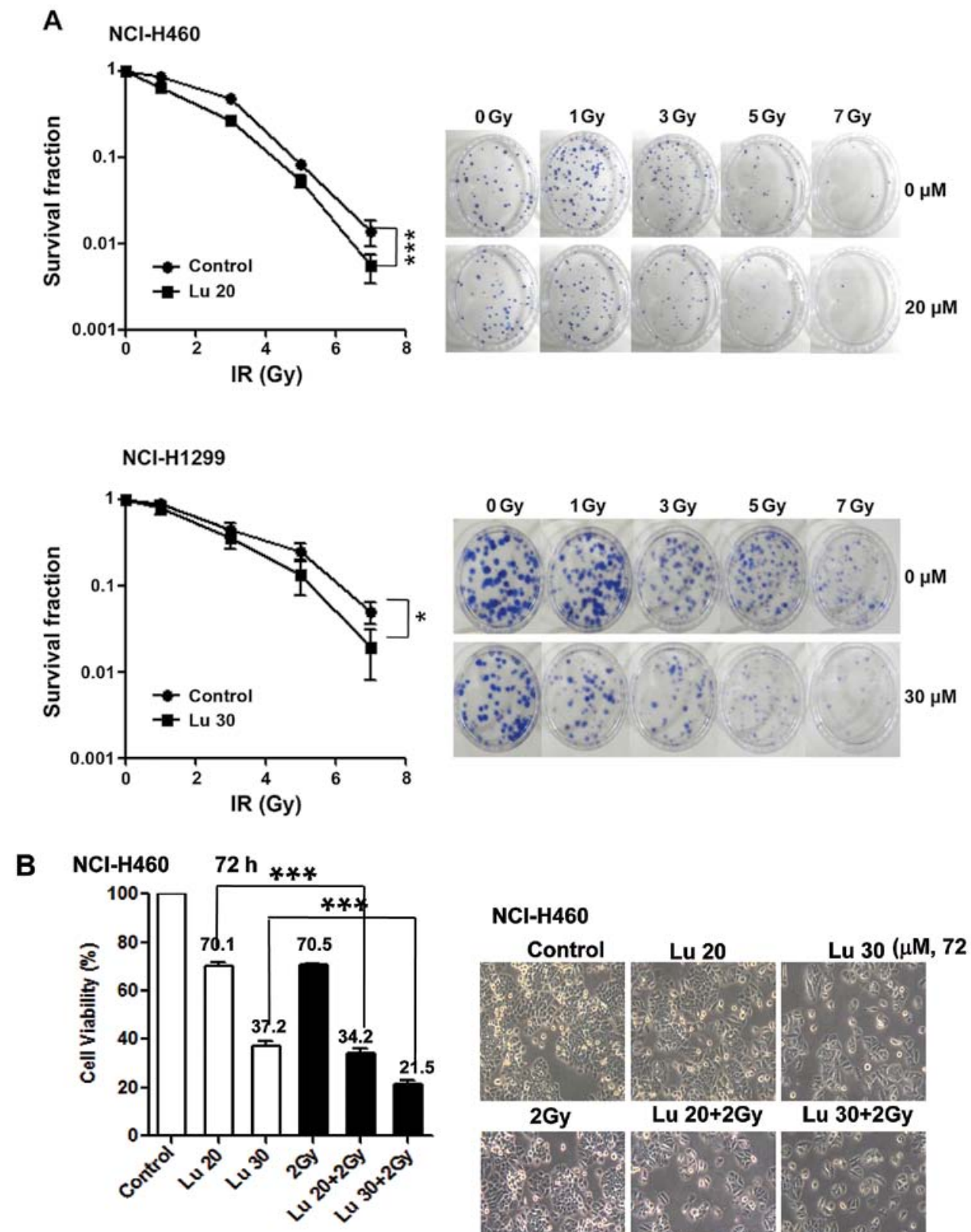

$\mathrm{NCl}-\mathrm{H} 460$

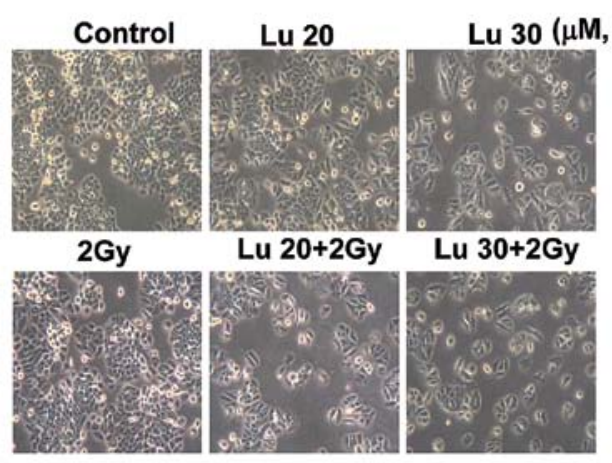

NCI-H1299

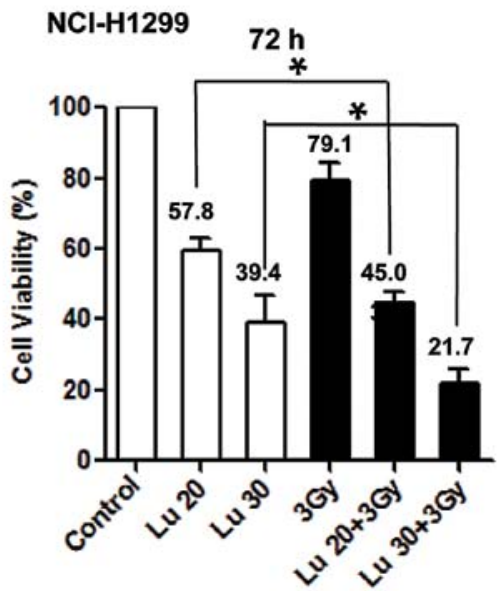

Control Lu $20 \quad$ Lu $30(\mu \mathrm{M}, 72 \mathrm{~h})$
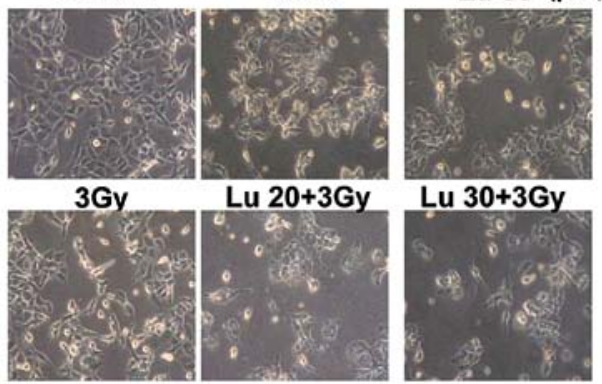

Lu 30+3Gy

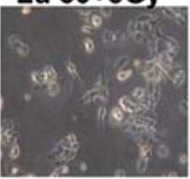

Figure 2. Combined treatment with 3',4',5',7'-tetrahydroxyflavone (luteolin) and ionizing radiation (IR) enhances cell death. (A) Clonogenic assays for NCI-H460 and -H1299 cells treated with the combination of luteolin and IR. (B) Cell counting assay for NCI-H460 and -H1299 cells treated with the combination of luteolin and IR. Lu, luteolin; control, mock-treated control; Lu 20 and 30, groups treated with 20 and $30 \mu \mathrm{M}$ luteolin only, respectively; 2 and $3 \mathrm{~Gy}$, groups treated with IR only; Lu 20/30 + 2 Gy/3 Gy, combinations of 20 or $30 \mu \mathrm{M}$ luteolin and 2 or 3 Gy of IR. ${ }^{*} \mathrm{P}<0.05$ and ${ }^{* * * *} \mathrm{p}<0.001$. 

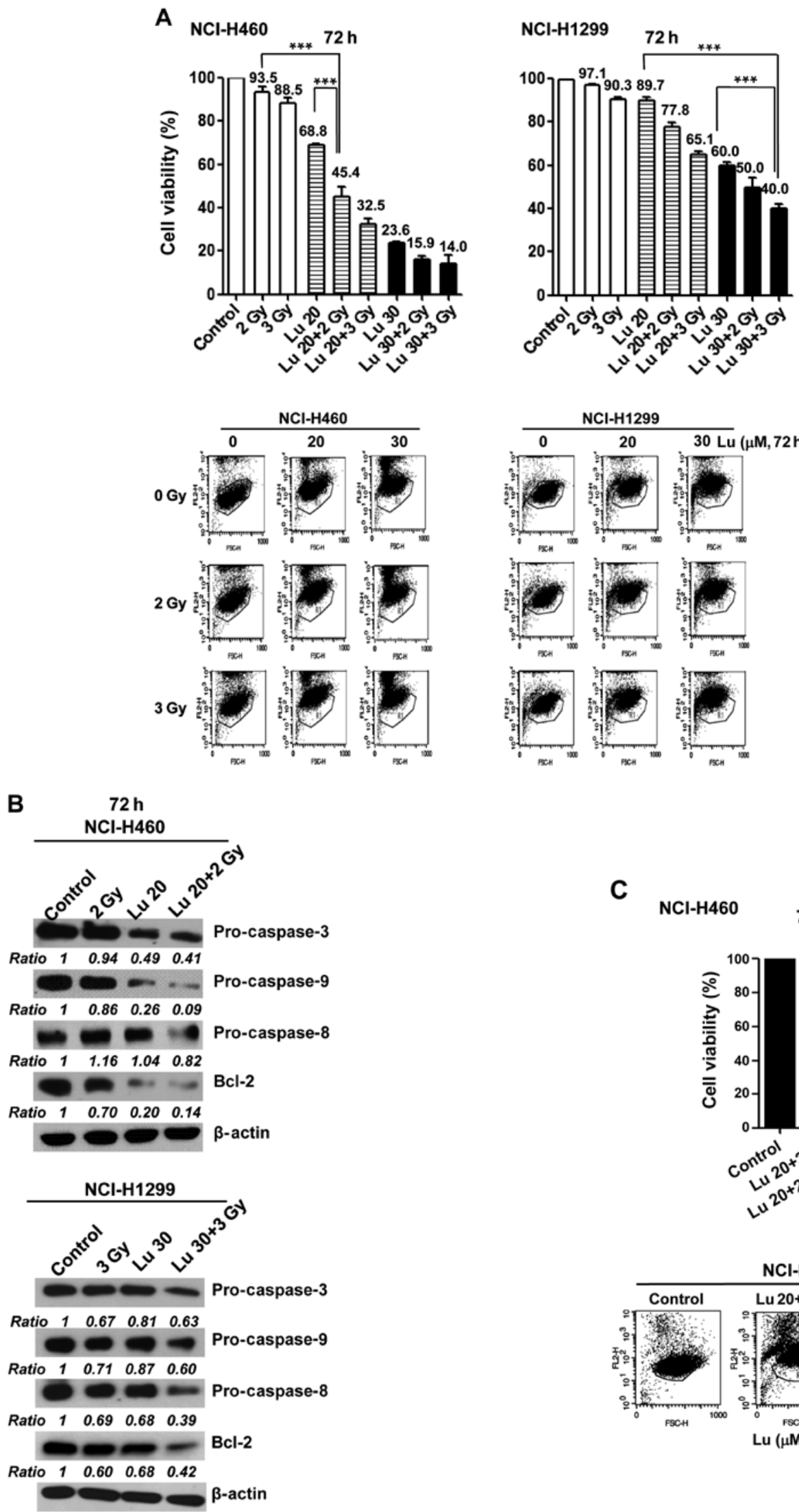

C

$\mathrm{NCl}-\mathrm{H} 460 \quad 72 \mathrm{~h}$
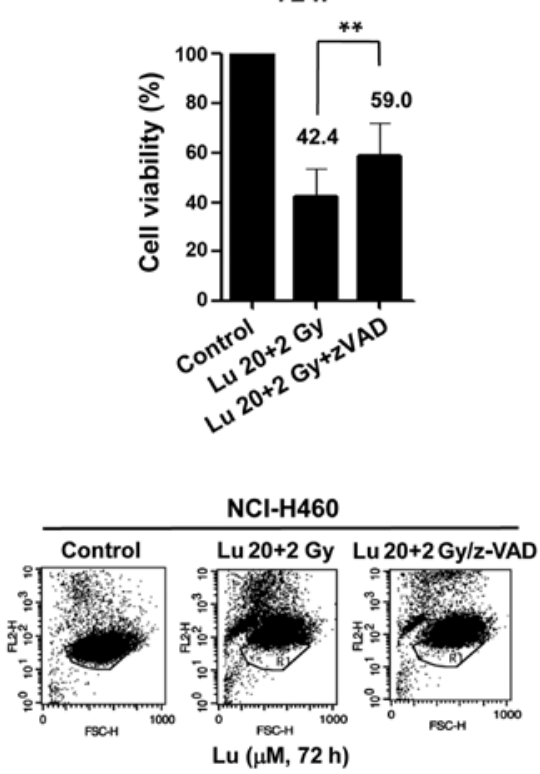

Figure 3. Combined treatment with 3',4',5',7'-tetrahydroxyflavone (luteolin) and ionizing radiation (IR) enhances apoptotic cell death in vitro. (A) Propidium iodide (PI) uptake assay for NCI-H460 and -H1299 cells receiving mock treatment (control) or treated with luteolin only, IR only, or a combination of luteolin and IR. Control, mock-treated control; Lu 20 and 30, groups treated with 20 and $30 \mu \mathrm{M}$ luteolin only, respectively; 2 and 3 Gy, groups treated with IR only; $\mathrm{Lu} 20 / 30+2 \mathrm{~Gy} / 3 \mathrm{~Gy}$, combinations of 20 or $30 \mu \mathrm{M}$ of luteolin and 2 or $3 \mathrm{~Gy}$ of IR. (B) Immunoblot detection of caspase-3, -8 and -9 , and B-cell lymphoma 2 (Bcl-2). NCI-H460 cells were mock-treated (control) or were treated with $20 \mu \mathrm{M}$ luteolin only, $2 \mathrm{~Gy} \mathrm{IR}$ only, or the combination of $20 \mu \mathrm{M}$ luteolin and $2 \mathrm{~Gy}$ IR. NCI-H1299 cells were mock-treated (control) or were treated with $30 \mu \mathrm{M}$ luteolin only, 3 Gy IR only, or the combination of $30 \mu \mathrm{M}$ luteolin and 3 Gy IR. (C) PI uptake assay for NCI-H460 cells treated with a combination of luteolin and IR with or without pre-treatment with $20 \mu \mathrm{M}$ carbobenzoxy-valyl-alanyl-aspartyl[O-methyl]-fluoromethylketone (z-VAD-fmk). Samples were harvested after treating for $72 \mathrm{~h}$. ${ }^{* *} \mathrm{P}<0.01$ and ${ }^{* * * *} \mathrm{p}<0.001$. 


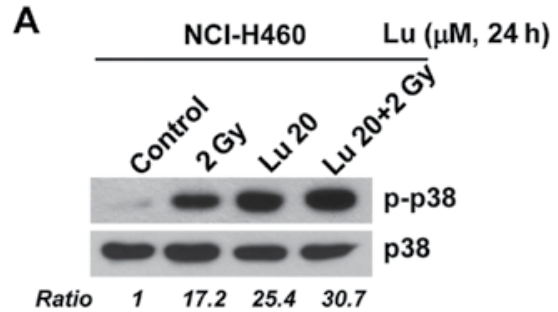

B


C



D

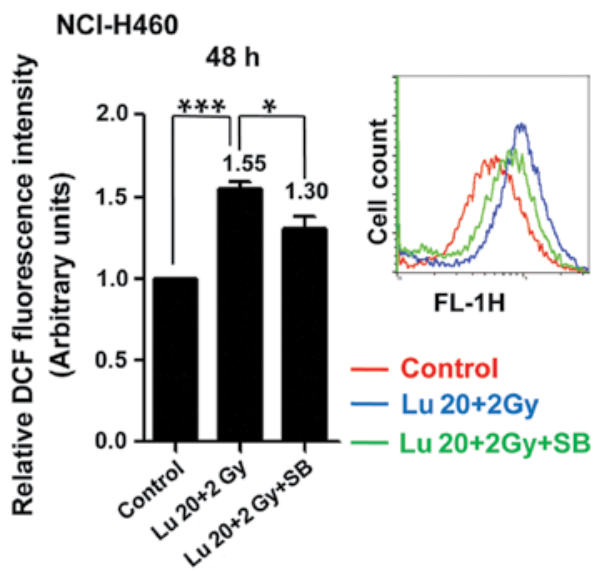

Figure 4. Apoptotic death of NCI-H460 cells induced by combined treatment with $3^{\prime}, 4^{\prime}, 5^{\prime}, 7^{\prime}$-tetrahydroxyflavone (luteolin) and ionizing radiation (IR) is mediated by p38. (A) Immunoblot detection of phosphorylated p38 mitogen-activated protein kinase (MAPK) in NCI-H460 cells receiving mock treatment (control) or treated with $20 \mu \mathrm{M}$ luteolin only, 2 Gy IR only, or a combination of $20 \mu \mathrm{M}$ luteolin and $2 \mathrm{~Gy} \mathrm{IR}$. Samples were harvested after $24 \mathrm{~h}$. (B) Propidium iodide (PI) uptake assay to determine the role of p38 MAPK in mediating apoptosis induced by the combination of $20 \mu \mathrm{M}$ luteolin and 2 Gy IR. Samples were pre-treated with SB205358 (SB) $(10 \mu \mathrm{M})$ or vehicle [dimethyl sulfoxide (DMSO)] for $1 \mathrm{~h}$ and then treated with the combination of $20 \mu \mathrm{M}$ luteolin and $2 \mathrm{~Gy}$ IR Samples were collected after $72 \mathrm{~h}$. (C) Immunoblot assay to detect caspase- 8 and -9 induced by the combination of $20 \mu \mathrm{M}$ luteolin and $2 \mathrm{~Gy}$ IR, with or without SB pre-treatment. Samples were pre-treated with SB $(10 \mu \mathrm{M})$ or vehicle (DMSO) for $1 \mathrm{~h}$ and then treated with the combination of $20 \mu \mathrm{M}$ luteolin and $2 \mathrm{~Gy}$ IR. Samples were collected after $72 \mathrm{~h}$. (D) Detection of reactive oxygen species (ROS) production induced by the combination of $20 \mu \mathrm{M}$ luteolin and $2 \mathrm{~Gy}$ IR, with or without SB pre-treatment $(10 \mu \mathrm{M})$ for $1 \mathrm{~h}$. Samples were harvested $72 \mathrm{~h}$ after treatment. ${ }^{*} \mathrm{P}<0.05$ and ${ }^{* * * *} \mathrm{p}<0.001$.

Accordingly, we determined $\mathrm{IC}_{50}$ values of luteolin against NSCLC cells using MTT assays. The $\mathrm{IC}_{50}$ values were determined to be $20.706 \mu \mathrm{M}$ in NCI-H460 cells and $25.291 \mu \mathrm{M}$ in NCI-H1299 cells (Fig. 1B), showing that luteolin alone is capable of killing NSCLC cells, as previously reported (18). To test the radiosensitizing effect of luteolin, we pre-treated NCI-H460 and -H1299 cells with 20 or $30 \mu \mathrm{M}$ luteolin for $6 \mathrm{~h}$, and then exposed cells to different doses of IR $(1,3$, 5 or 7 Gy). Clonogenic (Fig. 2A) and cell counting (Fig. 2B) assays confirmed the radiosensitizing effect of luteolin. Clonogenic assays showed that the survival fraction in the combination treatment group decreased compared with that in the IR-only treatment group. At a survival fraction of 0.25 , the DER value was calculated to be 1.22 and 1.35 for NCI-H460 and -H1299 cells, respectively (Fig. 2A). Cell counting assays also showed that the combination of luteolin $(20$ or $30 \mu \mathrm{M})$ and IR ( 2 or 3 Gy) enhanced cell death (Fig. 2B). Differences in the mean survival rates of NCI-H460 cells between the luteolin-only and combination treatment group ( 2 Gy IR) were $\sim 36 \%$ at $20 \mu \mathrm{M}$ luteolin and $\sim 15 \%$ at $30 \mu \mathrm{M}$ luteolin (Fig. 2B, upper panel), indicating that the combination of $20 \mu \mathrm{M}$ luteolin and 2 Gy IR was most effective in these cells. For NCI-H1299 cells (Fig. 2B, lower panel), differences in the mean survival rates between the luteolin-only and combination group (3 Gy IR) were $\sim 12 \%$ at $20 \mu \mathrm{M}$ luteolin and $\sim 17 \%$ at $30 \mu \mathrm{M}$ luteolin, indicating that the combination of $30 \mu \mathrm{M}$ luteolin and $3 \mathrm{~Gy}$ IR was most effective in this cell line. Collectively, these results indicate that luteolin acts as a radiosensitizer against NSCLCs and exerts a much stronger radiosensitizing effect in NCI-H460 cells.

Combined treatment with luteolin and IR enhances apoptotic cell death. Next, we tested which cell death pathway mediated the radiosensitizing effect of luteolin. NCI-H460 and -H1299 cells were pre-treated with 20 or $30 \mu \mathrm{M}$ luteolin for $6 \mathrm{~h}$, and then irradiated with 2 or 3 Gy IR. After $72 \mathrm{~h}$, cells were harvested and PI uptake was analyzed (Fig. 3A). In NCI-H460 cells, the combination of $20 \mu \mathrm{M}$ luteolin and $2 \mathrm{~Gy}$ IR increased apoptotic cell death by $\sim 48 \%$ compared with 2 Gy IR only and by $\sim 23 \%$ vs. $20 \mu \mathrm{M}$ luteolin only (Fig. 3A, left panel). In NCI-H1299 cells, the combination of $30 \mu \mathrm{M}$ luteolin and $3 \mathrm{~Gy}$ IR increased apoptotic cell death by 50\% compared with 3 Gy IR only, and by $\sim 20 \%$ vs. $30 \mu \mathrm{M}$ luteolin only (Fig. 3A, right panel). Immunoblot analyses also showed that these 
A

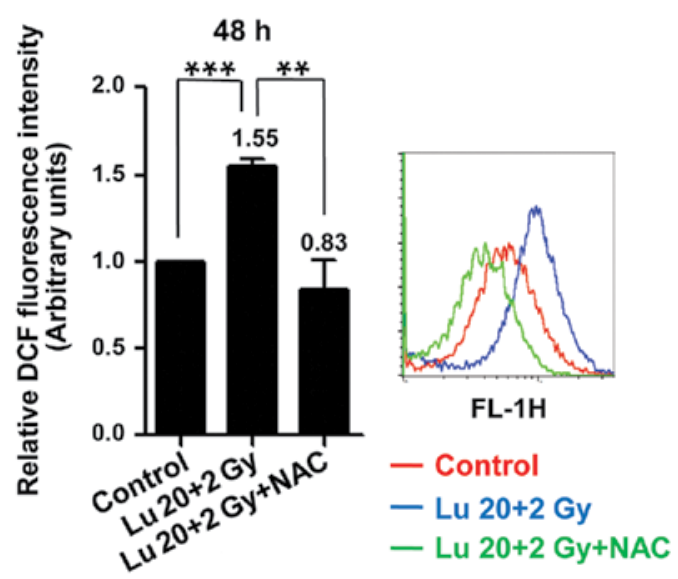

B

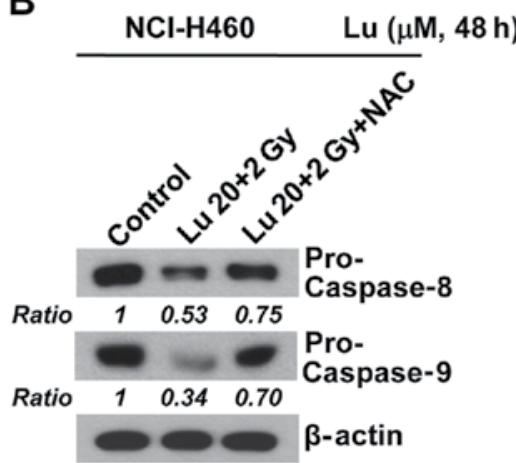

C

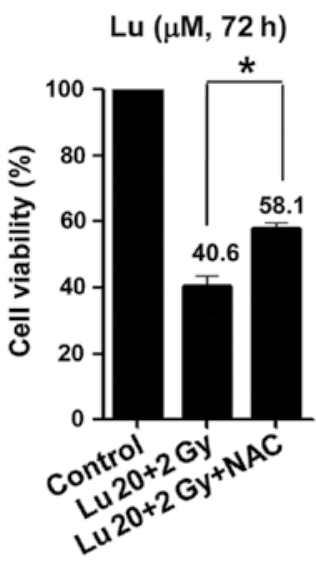

$\mathrm{NCl}-\mathrm{H} 460$

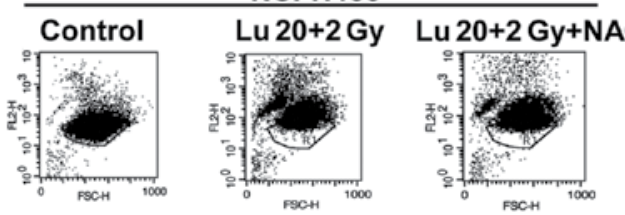

D

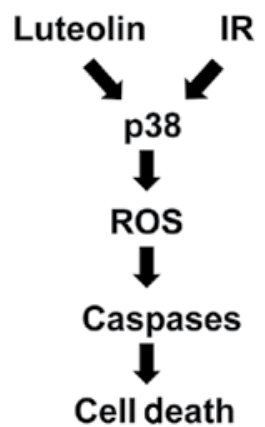

Figure 5. p38 mitogen-activated protein kinase (MAPK)-dependent reactive oxygen species (ROS) production mediates apoptotic cell death induced by combined treatment with 3',4',5',7'-tetrahydroxyflavone (luteolin) and ionizing radiation (IR). (A) Detection of ROS production induced by the combination of $20 \mu \mathrm{M}$ luteolin and $2 \mathrm{~Gy}$ IR. NCI-H460 cells were pre-incubated with or without $5 \mathrm{mM} \mathrm{N}$-acetyl-L-cysteine (NAC), and then treated with the combination of $20 \mu \mathrm{M}$ luteolin and $2 \mathrm{~Gy}$ IR for $48 \mathrm{~h}$. (B) Immunoblot detection of caspase-8 and -9 induced by the combination of $20 \mu \mathrm{M}$ luteolin and $2 \mathrm{~Gy}$ IR, with or without pre-incubation with $5 \mathrm{mM}$ NAC for $48 \mathrm{~h}$. (C) Propidium iodide (PI) uptake assay to detect apoptotic cell death under conditions in which ROS production was inhibited. NCI-H460 cells were pre-incubated with or without NAC, and then treated with the combination of $20 \mu \mathrm{M}$ luteolin and $2 \mathrm{~Gy}$ IR. (D) Scheme showing induction of the $\mathrm{p} 38 / \mathrm{ROS} / \mathrm{caspase}$ cascade by the combination of luteolin and IR. ${ }^{*} \mathrm{P}<0.05,{ }^{* *} \mathrm{p}<0.01$ and ${ }^{* * * *} \mathrm{p}<0.001$.

combination-treatment conditions $(20 \mu \mathrm{M}$ luteolin +2 Gy IR in NCI-H460 and $30 \mu \mathrm{M}$ luteolin +3 Gy IR in NCI-H1299) promoted activation of caspase-3, -8 and -9 , and decreased Bcl-2 levels (Fig. 3B). Pre-treatment with z-VAD-fmk, a chemical pan-caspase inhibitor, suppressed apoptotic death of NCI-H460 cells induced by combination treatment with luteolin and IR (Fig. 3C).

Phosphorylation of p38 increases ROS production and apoptotic cell death under conditions of combined treatment with luteolin and IR. We next examined modulation of apoptosis-related mitogen-activated protein kinase (MAPK) proteins in NCI-H460 cells by immunoblot analysis, and found that the combination of $20 \mu \mathrm{M}$ luteolin and 2 Gy IR induced p38 phosphorylation (Fig. 4A). Pre-treatment with SB250358, a specific chemical inhibitor of p38, attenuated apoptotic cell death induced by combination treatment, decreasing the percentage of apoptotic cells by $\sim 18 \%$ (Fig. 4B). Blockade of p38 also suppressed activation of caspases (Fig. 4C). Moreover, combined treatment induced ROS production, increasing ROS levels by $\sim 55 \%$ compared with controls. Interestingly, inhibition of p38 with SB250358 also blocked ROS production (Fig. 4D). Collectively, these results suggest that p38 might be a major signaling mediator of the radiosensitizing effects of luteolin through its effects on ROS production and apoptotic cell death.

Increased ROS production induced by combined treatment with luteolin and IR mediates apoptotic cell death in vitro. To examine the functional linkage between the p38-dependent generation of ROS and apoptosis, we tested the effects of the ROS scavenger NAC on the caspase activation and apoptosis induced by combined treatment with luteolin and IR. Pre-treatment of NCI-H460 cells with NAC caused a decrease in ROS production induced by combination treatment (Fig. 5A) in association with inhibition of caspase activations (Fig. 5B) and a decrease ( $17 \%)$ in apoptotic cell death (Fig. 5C). These results indicate that activation of a $\mathrm{p} 38 / \mathrm{ROS} / \mathrm{caspase}$ cascade might enhance apoptotic cell death and constitute a major intracellular signaling pathway for the radiosensitizing effects of luteolin (Fig. 5D). 
Combined treatment with luteolin and IR enhances apoptotic cell death in vivo. On the basis of the above in vitro results, we tested the radiosensitizing effects of luteolin in vivo using an NCI-H460 cell tumor xenograft model, measuring the time for tumors in each group to reach a volume of $1,500 \mathrm{~mm}^{3}$. Compared with controls, combination treatment resulted in a tumor growth delay of 21.8 days, yielding an enhancement factor of 1.83 (Fig. 6A and Table I). These results suggest that luteolin enhances radiation-induced cell death both in vitro and in vivo. Tumor tissue was excised from mice in each treatment group, as described in Materials and methods, and TUNEL assays were performed (Fig. 6B). Apoptotic cells were counted and plotted as a percentage of the total cell population. The number of apoptotic cells in the combination treatment group was $\sim 3$-fold higher than that in the IR-only group, and $\sim 5$-fold higher than that in the luteolin-only group. These results clearly suggest that the combination of luteolin and IR enhances cell death by enhancing apoptosis in vivo as well as in vitro.

\section{Discussion}

In the present study, we demonstrate that luteolin exerts radiosensitizing effects that enhance apoptotic cell death in NSCLC cells both in vitro and in vivo, and further show that these effects are mediated by activation of a p38/ROS/caspase cascade. First, we observed that the combination of luteolin and IR enhanced death of both NCI-H460 and -H1299 cells (Fig. 1). Several previous reports have shown that both $p 53$ and phosphatase and tensin homolog (PTEN) are major tumor suppressors that regulate drug and radiation responses $(19,20)$. However, because the NCI-H460 cell line contains wild-type $p 53$ and $P T E N$ but the NCI-H1299 cell line is p53- and PTEN-null, our results indicate that enhancement of cell death by the combination of luteolin and IR is independent of intracellular $p 53$ and PTEN status (21). Second, we found based on PI uptake that the combination of luteolin and IR enhances apoptotic cell death (Fig. 3A). Consistent with this, Bcl-2 protein levels were decreased and activation of caspase- $3,-8$, and -9 was increased by combination treatment (Fig. 3B). Numerous apoptosis-related proteins, including p53, Bcl-2-associated X protein (Bax), p21, Bcl-2 and caspases, are involved in the radiation-induced apoptotic death of cancer cells (22). These proteins might also be responsible for radiosensitizing effects and could be targets for the development of radio-enhancing reagents. Therefore, we could conclude that radiosensitizing effect of luteolin is dependent on apoptosis.

There are two major pathways of apoptosis: intrinsic and extrinsic (or death receptor-mediated). Activation of the intrinsic pathway is induced by external stress and is followed by changes in mitochondrial permeability transition (MPT) and activation of caspase-9. The extrinsic pathway begins with death receptor/ligand binding and proceeds through caspase- 8 activation. Caspase activation is a common event in both apoptotic pathways. Caspase- 8 and -9 are 'initiator' caspases of the extrinsic and intrinsic pathway, respectively, whereas caspase-3 is a common 'executioner' caspase in all apoptotic pathways. Bcl-2 inhibits apoptosis by regulating MPT and thereby blocking cytochrome $c$ release from mitochondria. Because the combination of luteolin and IR increased activation of both
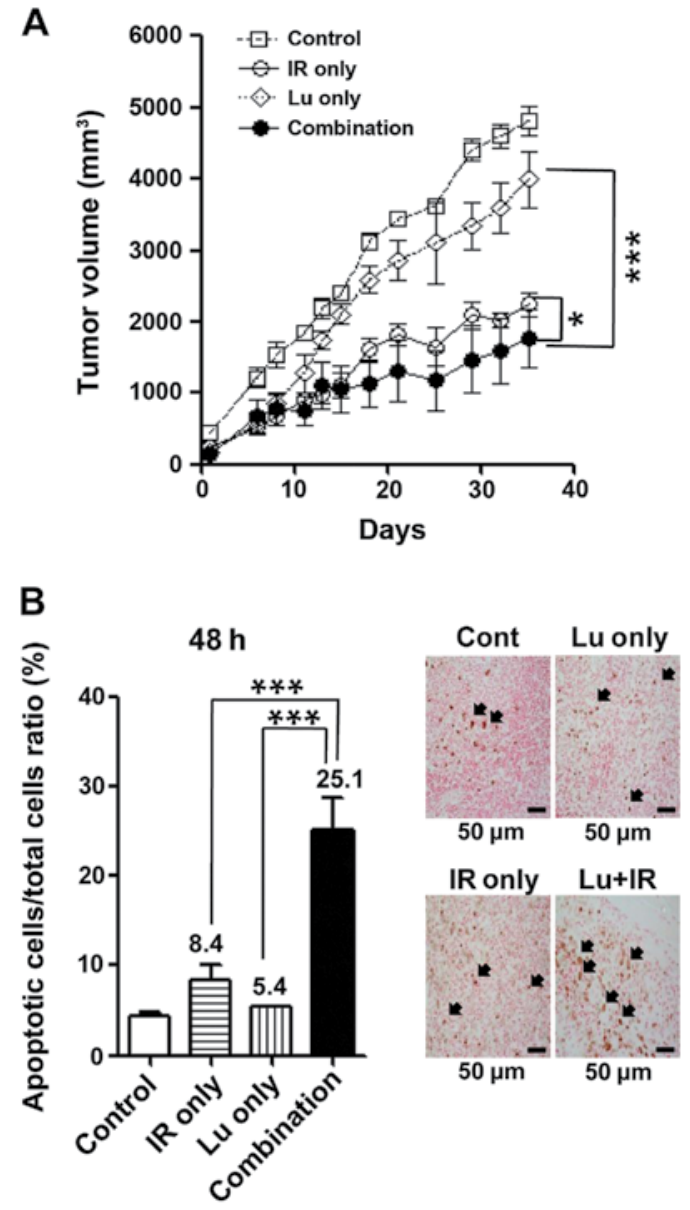

Figure 6. Combined treatment with $3^{\prime}, 4^{\prime}, 5^{\prime}, 7^{\prime}$-tetrahydroxyflavone (luteolin) and ionizing radiation (IR) enhances cell death in vivo. (A) An NCI-H460 cell xenograft model in nude mice was created and mice were divided into the following four groups ( $\mathrm{n}=5 \mathrm{mice} / \mathrm{group})$ : control (mock-treated), IR only (treated with 5 Gy IR only), Lu only (treated with $10 \mathrm{mg} / \mathrm{kg}$ luteolin only), and $\mathrm{Lu}+\mathrm{IR}$ (treated with both $10 \mathrm{mg} / \mathrm{kg}$ luteolin and $5 \mathrm{~Gy}$ IR), as described in Materials and methods. (B) Analysis of xenograft tumor tissue by terminal deoxynucleotidyl transferase dUTP nick end labeling (TUNEL) assay. Left panel: quantitative results of TUNEL assays; the number above each bar denotes the mean value for TUNEL assays performed in duplicate. Right panel: tissue section images showing TUNEL-stained apoptotic cells (arrows). ${ }^{*} \mathrm{P}<0.05$ and ${ }^{* * *} \mathrm{p}<0.001$

the intrinsic and extrinsic apoptotic caspases and decreased $\mathrm{Bcl}-2$ protein levels, we postulate that the radiosensitizer action of luteolin results from regulation of caspase activation and MPT (23). In addition to these apoptotic proteins, various intracellular proteins, including phosphatidylinositol 3-kinase, Akt, cell cycle-related molecules and elements of the DNA repair system, serve as targets of radiosensitizers. Radiosensitizing reagents that target these proteins can enhance radiation-induced cell death by perturbing various physiological phenomena-inhibiting angiogenesis, arresting or disrupting the cell cycle, inducing apoptosis, or blocking cell survival signaling pathways (24).

Experiments were performed to identify the intracellular signaling pathway involved in mediating the cell death enhancement induced by combined treatment with luteolin and IR implicated activation of a p38/ROS/caspase cascade (Figs. 4 and 5). Phosphorylation of p38 MAPK, one of three kinases that form the core of a MAPK cascade, was 
increased by the combination of luteolin and IR. MAP kinase kinase kinase (MAPKKK, also known as MEKK), the first of the three kinases, is located downstream of the original signaling protein, such as small GTPase, and phosphorylates the second kinase, MAP kinase kinase (MAPKK, also known as MEK or MKK) in this kinase cascade. Activated MAPKK isoforms, including MKK3, 4 and 6, then phosphorylate the last kinase of the cascade including $\mathrm{p} 38$. Several previous studies have shown that p38 MAPK is a stress-response molecule and involved in cell death by radiation and radiosensitizers. It has been reported that activation of c-Abl-PKCD-Rac1-p38 MAPK signaling by IR induces conformational changes in Bak and Bax, resulting in mitochondrial activation-mediated apoptotic cell death in human NSCLC cells (25). We also previously reported that the combination of a small chemical molecule and IR enhances cancer cell death through p38-mediated Bcl-2 degradation (26). In addition, we previously showed that IR activates c-Jun N-terminal kinase (JNK), another MAPK, and that activated JNK induces ROS production by disrupting mitochondrial membrane potential (17). Here, we found that p38 MAPK activation induced ROS production, which enhanced apoptotic cell death. ROS generated by IR damages cells through oxidation of lipids, DNA, and proteins. ROS production is mediated primarily by membrane-associated enzymes, such as NADPH oxidase, or is triggered intracellularly in the mitochondria through the electron transport chain (27). It is also known that oxidative damage caused by IR-induced ROS promotes apoptosis through activation of caspases. Park et al (28) reported that the combination of phytosphingosine and IR enhanced apoptosis via ROS-induced mitochondrial relocalization of Bax and nuclear translocation of apoptosis-inducing factor (AIF). Lee et al (29) also reported that IR can act through protein kinase $\mathrm{C}-\delta(\mathrm{PKC} \delta)$-mediated ROS production to induce apoptosis. Therefore, the enhancement of apoptosis by the combination of luteolin and IR through activation of p38/ROS/caspases reported here is in accord with the previous reports $(28,29)$. However, inhibition of p38/ROS/caspase did not complete abrogate the cell death induced by combined treatment with luteolin and IR. This implies that other cell death or growth-retardation mechanisms might modulate the radiosensitizing effect of luteolin, raising interesting questions for further research.

Taken together, our results suggest a novel role for luteolin as a radiosensitizer in NSCLC cells, where it acts by increasing apoptotic cell death by the activation of the p38/ROS/caspase cascade, and it is independent of the expression of p53 and PTEN. Development of an ideal radiosensitizer must consider two aspects, increased efficiency and protection of normal tissues (10). We did not determine if luteolin protected normal tissue from damage by IR, but the combination of luteolin and IR enhanced cancer cell death in vivo as well as in vitro. The radiosensitization of cells by the combination of luteolin and IR shares a common stress-response signaling pathway containing p38 MAPK and ROS $(30,31)$. Elucidation of the exact mechanism of radiosensitization is important in order to develop new drugs that are synergistic with IR. Our findings on the radiosensitizing effects of luteolin may be useful to develop therapeutic techniques for the treatment of NSCLC patients regardless of p53 and PTEN expression status.

\section{Acknowledgements}

This study was supported by the Nuclear Research and Development Program of the National Research Foundation of Korea (NRF) (Seoul, Korea) grant funded by the Korean government (MEST) (2012M2A2A7010459) and, in part, by the Basic Science Research Program through the NRF (2008-0062611).

\section{References}

1. Jemal A, Siegel R, Ward E, Hao Y, Xu J, Murray T and Thun MJ: Cancer Statistics, 2008. CA Cancer J Clin 58: 71-96, 2008.

2. Kim JS, Amorino GP, Pyo H, Cao Q, Price JO and Choy H: The novel taxane analogs, BMS-184476 and BMS-188797, potentiate the effects of radiation therapy in vitro and in vivo against human lung cancer cells. Int J Radiat Oncol Biol Phys 51: 525-534, 2001.

3. Palayoor ST, Bump EA, Calderwood SK, Bartol S and Coleman CN: Combined antitumor effect of radiation and ibuprofen in human prostate carcinoma cells. Clin Cancer Res 4: 763-771, 1998.

4. Dicker AP, Williams TL and Grant DS: Targeting angiogenic processes by combination rofecoxib and ionizing radiation. Am J Clin Oncol 24: 438-442, 2001.

5. Kim KY, Seol JY, Jeon GA and Nam MJ: The combined treatment of aspirin and radiation induces apoptosis by the regulation of bcl-2 and caspase-3 in human cervical cancer cells. Cancer Lett 189: 157-166, 2003.

6. Zhu AX and Willett CG: Chemotherapeutic and biologic agents as radiosensitizers in rectal cancer. Semin Radiat Oncol 13: 454-468, 2003.

7. Jones PD, de Lorimier LP, Kitchell BE and Losonsky JM: Gemcitabine as a radiosensitizer for nonresectable feline oral squamous cell carcinoma. J Am Anim Hosp Assoc 39: 463-467, 2003.

8. Begg AC, Stewart FA and Vens C: Strategies to improve radiotherapy with targeted drugs. Nat Rev Cancer 11: 239-253, 2011

9. Mitsudomi T, Suda K and Yatabe Y: Surgery for NSCLC in the era of personalized medicine. Nat Rev Clin Oncol 10: 235-244, 2013.

10. Moding EJ, Kastan MB and Kirsch DG: Strategies for optimizing the response of cancer and normal tissues to radiation. Nat Rev Drug Discov 12: 526-542, 2013.

11. Travis WD, Brambilla E, Noguchi M, et al: International Association for the Study of Lung Cancer/American Thoracic Society/European Respiratory Society: international multidisciplinary classification of lung adenocarcinoma: executive summary. Proc Am Thorac Soc 8: 381-385, 2011.

12. Swinney DC and Anthony J: How were new medicines discovered? Nat Rev Drug Discov 10: 507-519, 2011.

13. Rask-Andersen M, Almén MS and Schiöth HB: Trends in the exploitation of novel drug targets. Nat Rev Drug Discov 10: 579-590, 2011.

14. Seelinger G, Merfort I, Wölfle U and Schempp CM: Anti-carcinogenic effects of the flavonoid luteolin. Molecules 13: 2628-2651, 2008

15. Lin Y, Shi R, Wang X and Shen HM: Luteolin, a flavonoid with potential for cancer prevention and therapy. Curr Cancer Drug Targets 8: 634-646, 2008.

16. Albert JM, Cao C, Kim KW, et al: Inhibition of poly(ADP-ribose) polymerase enhances cell death and improves tumor growth delay in irradiated lung cancer models. Clin Cancer Res 13: 3033-3042, 2007.

17. Kim EM, Yang HS, Kang SW, Ho JN, Lee SB and Um HD: Amplification of the gamma-irradiation-induced cell death pathway by reactive oxygen species in human U937 cells. Cell Signal 20: 916-924, 2008.

18. Leung HW, Kuo CL, Yang WH, Lin CH and Lee HZ: Antioxidant enzymes activity involvement in luteolin-induced human lung squamous carcinoma CH27 cell apoptosis. Eur J Pharmacol 534: 12-18, 2006.

19. Rebucci M and Michiels C: Molecular aspects of cancer cell resistance to chemotherapy. Biochem. Pharmacol 85: 1219-1226, 2013.

20. Song G, Ouyang $\mathrm{G}$ and Bao S: The activation of Akt/PKB signaling pathway and cell survival. J Cell Mol Med 9: 59-71, 2005. 
21. Park JK, Jung HY, Park SH, Kang SY, Yi MR, Um HD and Hong SH: Combination of PTEN and gamma-ionizing radiation enhances cell death and $\mathrm{G}(2) / \mathrm{M}$ arrest through regulation of AKT activity and p21 induction in non-small-cell lung cancer cells. Int J Radiat Oncol Biol Phys 70: 1552-1560, 2008.

22. Prise KM, Schettino G, Folkard M and Held KD: New insights on cell death from radiation exposure. Lancet Oncol 6: 520-528, 2005.

23. Elmore S: Apoptosis: a review of programmed cell death. Toxicol Pathol 35: 495-516, 2007.

24. Katz D, Ito E and Liu FF: On the path to seeking novel radiosensitizers. Int J Radiat Oncol Biol Phys 73: 988-996, 2009.

25. Munshi A and Ramesh R: Mitogen-activated protein kinases and their role in radiation response. Genes Cancer 4: 401-408, 2013.

26. Park JK, Chung YM, Kim BG, Yoo YA, Yang BS, Kim JS and Yoo YD: N'-(phenyl-pyridin-2-yl-methylene)-hydrazine carbodithioic acid methyl ester enhances radiation-induced cell death by targeting Bcl-2 against human lung carcinoma cells. Mol Cancer Ther 3: 403-407, 2004.
27. Caputo F, Vegliante R and Ghibelli L: Redox modulation of the DNA damage response. Biochem Pharmacol 84: 1292-1306, 2012.

28. Park MT, Kim MJ, Kang YH, et al: Phytosphingosine in combination with ionizing radiation enhances apoptotic cell death in radiation-resistant cancer cells through ROS-dependent and -independent AIF release. Blood 105: 1724-1733, 2005.

29. Lee YJ, Lee DH, Cho CK, et al: HSP25 inhibits radiation-induced apoptosis through reduction of PKCdelta-mediated ROS production. Oncogene 24: 3715-3725, 2005.

30. Wagner EF and Nebreda AR: Signal integration by JNK and p38 MAPK pathways in cancer development. Nat Rev Cancer 9: 537-549, 2009.

31. Ju W, Wang X, Shi H, Chen W, Belinsky SA and Lin Y: A critical role of luteolin-induced reactive oxygen species in blockage of tumor necrosis factor-activated nuclear factor-kappaB pathway and sensitization of apoptosis in lung cancer cells. Mol Pharmacol 71: $1381-1388,2007$. 